\title{
Experimental generation of tripartite polarization entangled states of bright optical beams
}

\author{
Liang Wu, ${ }^{1}$ Zhihui Yan, ${ }^{1,2}$ Yanhong Liu, ${ }^{1}$ Ruijie Deng, ${ }^{1}$ Xiaojun Jia,,${ }^{1}$, , a Changde Xie,,${ }^{1,2}$ and Kunchi \\ Peng ${ }^{1,2}$ \\ ${ }^{1)}$ State Key Laboratory of Quantum Optics and Quantum Optics Devices, Institute of Opto-Electronics, Shanxi University, Taiyuan, \\ 030006, People's Republic of China \\ ${ }^{2)}$ Collaborative Innovation Center of Extreme Optics, Shanxi University, Taiyuan 030006, \\ People's Republic of China
}

\begin{abstract}
The multipartite polarization entangled states of bright optical beams directly associating with the spin states of atomic ensembles are one of the essential resources in the future quantum information networks, which can be conveniently utilized to transfer and convert quantum states across a network composed of many atomic nodes. In this letter, we present the experimental demonstration of tripartite polarization entanglement described by Stokes operators of optical field. The tripartite entangled states of light at the frequency resonant with D1 line of Rubidium atoms are transformed into the continuous variable polarization entanglement among three bright optical beams via an optical beam splitter network. The obtained entanglement is confirmed by the extended criterion for polarization entanglement of multipartite quantized optical modes.
\end{abstract}

Quantum entanglement plays the kernel role in the developing of quantum information technology and has been applied in a variety of quantum communication and computation protocols $1-8$. At present, the interest has focused on building the quantum internet ${ }^{9}$ composed of quantum nodes and quantum information transmission channe10,11. Continuousvariable $(\mathrm{CV})$ polarization entanglement of optical field can be manipulated and detected with high efficiency and the bright polarization entangled beams can be directly measured without the need of a local oscillator ${ }^{12-15}$. Furthermore, both the polarization of light and atomic spin are described by Stokes operators, and the fluctuations of the polarization variables can be easily mapped onto the collective fluctuations of an atomic ensemble, thus the quantum state transfer between $\mathrm{CV}$ polarization states and spin states of atomic ensembles can be conveniently realized ${ }^{12}$. In 2002, N. V. Korolkova et al. introduced the physical concept about $\mathrm{CV}$ polarization entangled states and proposed schemes of generating and characterizing them 16 . P. K. Lam's group experimentally demonstrated CV polarization squeezing and bipartite entanglement by means of two degenerate optical parameter amplifiers (DOPAs) ${ }^{13}$. Then the polarization entanglement between two optical modes was realized by G. Leuchs's group with the asymmetric fiber-optic Sagnac interferometer ${ }^{14,17}$. The polarization entanglement was also produced in cold cesium atoms placed inside an optical cavity with high finesse ${ }^{18}$.

However, the bipartite entanglement is not enough to establish quantum networks, thus we have to prepare the polarization entangled states with more than two submodes. Here we report the experimental generation of $\mathrm{CV}$ tripartite polarization entangled states of light resonant on the $\mathrm{Rb} \mathrm{D} 1$ line (795 $\mathrm{nm}$ ), which are suitable for implementing optical storage and realizing the interaction between light and atoms. According to the inseparability criterion for the multipartite polarization entanglement deduced by us before ${ }^{19}$, the obtained polarization entangled state is characterized quantitatively. The exper-

\footnotetext{
${ }^{a)}$ Electronic mail: jiaxj@sxu.edu.cn
}

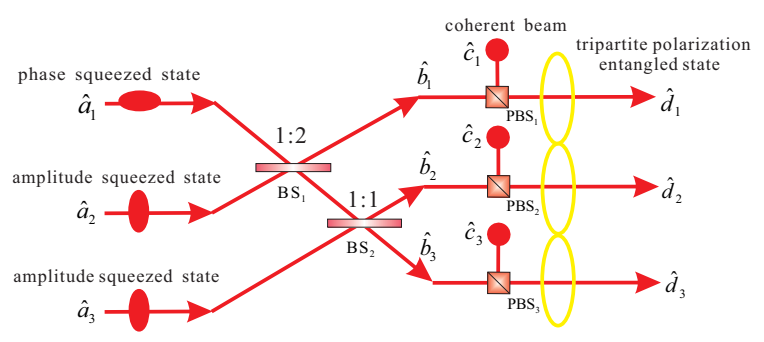

FIG. 1. Schematic for the generation of tripartite polarization entangled state.

imentally produced tripartite entangled states also satisfies the criterion for the genuine multipartite entanglement deduced by R. Y. Teh and M. D. Reid ${ }^{20}$. The experimental system and scheme can be directly extended to produce $\mathrm{CV}$ polarization entangled states with more submodes.

In quantum optics, the Stokes operators $\left(\hat{S}_{0}, \hat{S}_{1}, \hat{S}_{2}\right.$ and $\left.\hat{S}_{3}\right)$ are usually used to describe the polarization state of light 21 , which satisfy a spherical equation $\hat{S}_{1}^{2}+\hat{S}_{2}^{2}+\hat{S}_{3}^{2}=\hat{S}_{0}^{2}+2 \hat{S}_{0}$ and constitute a Poincaré sphere ${ }^{15,16}$. Where $\hat{S}_{0}$ represents the beam intensity whereas $\hat{S}_{1}, \hat{S}_{2}$ and $\hat{S}_{3}$ characterize its polarization and form a Cartesian axes system, which can be easily mapped to the spin operators of the atomic media. The Stokes parameters for pure states can be described by the corresponding annihilation $\hat{a}_{H(V)}$ and creation $\hat{a}_{H(V)}^{\dagger}$ operators of the constituent horizontally (subscript $\mathrm{H}$ ) and vertically (subscript $\mathrm{V})$ polarized modes in the frequency space, that are:

$$
\begin{aligned}
& \hat{S}_{0}=\hat{a}_{H}^{\dagger} \hat{a}_{H}+\hat{a}_{V}^{\dagger} \hat{a}_{V}, \hat{S}_{2}=\hat{a}_{H}^{\dagger} \hat{a}_{V} e^{i \theta}+\hat{a}_{V}^{\dagger} \hat{a}_{H} e^{-i \theta}, \\
& \hat{S}_{1}=\hat{a}_{H}^{\dagger} \hat{a}_{H}-\hat{a}_{V}^{\dagger} \hat{a}_{V}, \hat{S}_{3}=i \hat{a}_{V}^{\dagger} \hat{a}_{H} e^{-i \theta}-i \hat{a}_{H}^{\dagger} \hat{a}_{V} e^{i \theta}
\end{aligned}
$$

Where $\theta$ is the relative phase between the $\mathrm{H}$ and $\mathrm{V}$ polarization modes.

The schematic for the generation of tripartite polarization entangled state is shown in Fig. 1. A quadrature phase squeezed state of light $\left(\hat{a}_{1}\right)$ and two quadrature amplitude 
squeezed state of light ( $\hat{a}_{2}$ and $\left.\hat{a}_{3}\right)$ interfere on a beam splitters $\mathrm{BS}_{1}\left(\mathrm{BS}_{2}\right)$, with the ratio of reflectivity and transmissivity $\mathrm{R}: \mathrm{T}=1: 2(1: 1)$ to generate a tripartite GHZ-like entangled state $\left(\hat{b}_{1}, \hat{b}_{2} \text { and } \hat{b}_{3}\right)^{22}$. The three submodes $\hat{b}_{1}, \hat{b}_{2}$ and $\hat{b}_{3}$, each of which is a weak horizonal polarized $(\mathrm{H})$ states, are coupled with three strong vertical polarized $(\mathrm{V})$ coherent beams $\hat{c}_{1}$, $\hat{c}_{2}$ and $\hat{c}_{3}$ on three polarization beam splitters $\left(\mathrm{PBS}_{1-3}\right)$, respectively. For simplicity and without loss of generality, the average power of the three squeezed states of light (coherent light) is adjusted to equal, that is: $\alpha_{a_{1}}^{2}=\alpha_{a_{2}}^{2}=\alpha_{a_{3}}^{2}=\alpha_{a}^{2}$ $\left(\alpha_{c_{1}}^{2}=\alpha_{c_{2}}^{2}=\alpha_{c_{3}}^{2}=\alpha_{c}^{2}\right)$. The ratio of the intensity of the squeezing and coherent light is: $\alpha_{a}^{2} / \alpha_{c}^{2}=1 / 30$. The phase difference $\theta$ on the beam splitters $\mathrm{BS}_{1,2}$ and $\mathrm{PBS}_{1-3}$ all are controlled to $2 k \pi$ ( $k$ is an integer). The noise operators of the quadrature amplitude $\delta \hat{X}_{a_{i}}^{+}(\Omega)$ (phase $\delta \hat{X}_{a_{i}}^{-}(\Omega)$ ) of a squeezed state at the sideband frequency $(\Omega)$ can be expressed as $\delta \hat{X}_{a_{2(3)}}^{+}(\Omega)=e^{-r_{2(3)}} \delta \hat{X}_{a_{2(3)}}^{+(0)}(\Omega), \delta \hat{X}_{a_{2(3)}}^{-}(\Omega)=$ $e^{r_{2(3)}+r_{2(3)}^{\prime}} \delta \hat{X}_{a_{2(3)}}^{-(0)}(\Omega) \quad\left(\delta \hat{X}_{a_{1}}^{+}(\Omega)=e^{r_{1}+r_{1}^{\prime}} \delta \hat{X}_{a_{1}}^{+(0)}(\Omega)\right.$, $\delta \hat{X}_{a_{1}}^{-}(\Omega)=e^{-r_{1}} \delta \hat{X}_{a_{1}}^{-(0)}(\Omega)$ ), where $\delta \hat{X}_{a_{i}}^{ \pm(0)}(\Omega)$ are the amplitude (+) and phase (-) noise operators of the input beams of DOPAs, $r_{i}$ is the squeezing parameter and $r_{i}^{\prime}$ is the factor of extra noise on the antisqueezed quadrature components ${ }^{23}$. Since $\alpha_{a}^{2} \ll \alpha_{c}^{2}$ the quantum fluctuation variance of Stokes operators are expressed as

$$
\begin{aligned}
& \delta^{2} \hat{S}_{0_{d_{1}\left(d_{2}, d_{3}\right)}}(\Omega)=\delta^{2} \hat{S}_{1_{d_{1}\left(d_{2}, d_{3}\right)}}(\Omega)=4 \alpha_{c}^{2} \delta^{2} \hat{X}_{c_{1(2,3)}}^{+}(\Omega) \\
& \delta^{2} \hat{S}_{2_{d_{1}}}(\Omega)=4 \alpha_{c}^{2}\left(\frac{e^{2 r_{1}+2 r_{1}^{\prime}}}{3} \delta^{2} \hat{X}_{a_{1}}^{+(0)}(\Omega)+\frac{2 e^{-2 r_{2}}}{3} \delta^{2} \hat{X}_{a_{2}}^{+(0)}(\Omega)\right) \\
& \delta^{2} \hat{S}_{3_{d_{1}}}(\Omega)=4 \alpha_{c}^{2}\left(\frac{e^{-2 r_{1}}}{3} \delta^{2} \hat{X}_{a_{1}}^{-(0)}(\Omega)+\frac{2 e^{2 r_{2}+2 r_{2}^{\prime}}}{3} \delta^{2} \hat{X}_{a_{2}}^{-(0)}(\Omega)\right) \\
& \delta^{2} \hat{S}_{2_{d_{2}}}(\Omega)=4 \alpha_{c}^{2}\left(\frac{e^{2 r_{1}+2 r_{1}^{\prime}}}{3} \delta^{2} \hat{X}_{a_{1}}^{+(0)}(\Omega)\right. \\
& \left.-\frac{e^{-2 r_{2}}}{6} \delta^{2} \hat{X}_{a_{2}}^{+(0)}(\Omega)+\frac{e^{-2 r_{3}}}{2} \delta^{2} \hat{X}_{a_{3}}^{+(0)}(\Omega)\right) \\
& \delta^{2} \hat{S}_{3_{d_{2}}}(\Omega)=4 \alpha_{c}^{2}\left(\frac{e^{-2 r_{1}}}{3} \delta^{2} \hat{X}_{a_{1}}^{-(0)}(\Omega)\right. \\
& \left.-\frac{e^{2 r_{2}+2 r_{2}^{\prime}}}{6} \delta^{2} \hat{X}_{a_{2}}^{-(0)}(\Omega)+\frac{e^{2 r_{3}+2 r_{3}^{\prime}}}{2} \delta^{2} \hat{X}_{a_{3}}^{-(0)}(\Omega)\right)
\end{aligned}
$$$$
\delta^{2} \hat{S}_{3_{d_{3}}}(\Omega)=4 \alpha_{c}^{2}\left(\frac{e^{2 r_{1}+2 r_{1}^{\prime}}}{3} \delta^{2} \hat{X}_{a_{1}}^{+(0)}(\Omega)\right.
$$$$
\left.-\frac{e^{-2 r_{2}}}{6} \delta^{2} \hat{X}_{a_{2}}^{+(0)}(\Omega)-\frac{e^{-2 r_{3}}}{2} \delta^{2} \hat{X}_{a_{3}}^{+(0)}(\Omega)\right),
$$$$
\delta^{2} \hat{S}_{2_{d_{3}}}(\Omega)=4 \alpha_{c}^{2}\left(\frac{e^{-2 r_{1}}}{3} \delta^{2} \hat{X}_{a_{1}}^{-(0)}(\Omega)\right.
$$$$
\left.-\frac{e^{2 r_{2}+2 r_{2}^{\prime}}}{6} \delta^{2} \hat{X}_{a_{2}}^{-(0)}(\Omega)-\frac{e^{2 r_{3}+2 r_{3}^{\prime}}}{2} \delta^{2} \hat{X}_{a_{3}}^{-(0)}(\Omega)\right) .
$$

Where $\delta^{2} \hat{S}_{j_{d_{k}}}(\Omega)(j=0,1,2,3 . k=1,2,3)$ are the variances of Stokes operators of beam $d_{k}, \delta^{2} \hat{X}_{c_{1(2,3)}}^{+}(\Omega)$ are the variances of quadrature amplitude operators of beam $c_{1-3}$.

W. P. Bowen et al. extended the inseparability criterion characterizing $\mathrm{CV}$ quadrature entanglement ${ }^{24,25}$ to $\mathrm{CV}$ bipar- tite polarization entanglement $\frac{13}{3}$. P. van Loock and A. Furusawa gave the inseparability criterion formula for multipartite states in $2003^{22}$. Very recently, we deduced the tripartite inseparability criterion of Stokes operators for optical beams $\frac{19}{}$ based on the theoretical analysis in Ref. [22]:

$$
\begin{aligned}
& I_{1} \equiv \frac{\delta^{2}\left(\hat{S}_{2_{d_{2}}}-\hat{S}_{2_{d_{3}}}\right)+\delta^{2}\left(g_{1} \hat{S}_{3_{d_{1}}}+\hat{S}_{3_{d_{2}}}+\hat{S}_{3_{d_{3}}}\right)}{4\left|\alpha_{c}^{2}-\alpha_{a}^{2}\right|} \geq 1, \\
& I_{2} \equiv \frac{\delta^{2}\left(\hat{S}_{2_{d_{1}}}-\hat{S}_{2_{d_{3}}}\right)+\delta^{2}\left(\hat{S}_{3_{d_{1}}}+g_{2} \hat{S}_{3_{d_{2}}}+\hat{S}_{3_{d_{3}}}\right)}{4\left|\alpha_{c}^{2}-\alpha_{a}^{2}\right|} \geq 1, \\
& I_{3} \equiv \frac{\delta^{2}\left(\hat{S}_{2_{d_{1}}}-\hat{S}_{2_{d_{2}}}\right)+\delta^{2}\left(\hat{S}_{3_{d_{1}}}+\hat{S}_{3_{d_{2}}}+g_{3} \hat{S}_{3_{d_{3}}}\right)}{4\left|\alpha_{c}^{2}-\alpha_{a}^{2}\right|} \geq 1 .
\end{aligned}
$$

Where, $I_{1}, I_{2}$ and $I_{3}$ are the normalized correlation variances among Stokes operators, $g_{j}(j=1,2,3)$ are the adjustable classical gains for minimizing the correlation variances. When any two in the three inequalities are simultaneously violated, the three optical modes are in a tripartite polarization inseparable state.

In order to show the dependence of the correlation variances on the experimental parameters, the expressions of the normalized tripartite correlation variances based on the experimental parameters of three optical submodes can be obtained:

$$
\begin{aligned}
I_{1}= & \left\{\alpha _ { c } ^ { 2 } \left[12 e^{-2 r_{3}}+2\left(g_{1}+2\right)^{2} e^{-2 r_{1}}+4\left(g_{1}-1\right)^{2}\right.\right. \\
& \left.\left.e^{2\left(r_{2}+r_{2}^{\prime}\right)}\right]\right\} /\left(24\left|\alpha_{c}^{2}-\alpha_{a}^{2}\right|\right), \\
I_{2}= & \left\{\alpha _ { c } ^ { 2 } \left[3 e^{-2 r_{3}}+9 e^{-2 r_{2}}+2\left(g_{2}+2\right)^{2} e^{-2 r_{1}}+3\left(g_{2}-1\right)^{2}\right.\right. \\
& \left.\left.e^{2\left(r_{3}+r_{3}^{\prime}\right)}+\left(g_{2}-1\right)^{2} e^{2\left(r_{2}+r_{2}^{\prime}\right)}\right]\right\} /\left(24\left|\alpha_{c}^{2}-\alpha_{a}^{2}\right|\right), \\
I_{3}= & \left\{\alpha _ { c } ^ { 2 } \left[3 e^{-2 r_{3}}+9 e^{-2 r_{2}}+2\left(g_{3}+2\right)^{2} e^{-2 r_{1}}+3\left(g_{3}-1\right)^{2}\right.\right. \\
& \left.\left.e^{2\left(r_{3}+r_{3}^{\prime}\right)}+\left(g_{3}-1\right)^{2} e^{2\left(r_{2}+r_{2}^{\prime}\right)}\right]\right\} /\left(24\left|\alpha_{c}^{2}-\alpha_{a}^{2}\right|\right) .
\end{aligned}
$$

Calculating the minimum values of the expressions (5), we get the dependence of the optimal gains $\left(g_{i}^{o p t}\right)$ on the experimental parameters, that are:

$$
\begin{aligned}
& g_{1}^{o p t}=\frac{2 e^{2 r_{1}+2 r_{2}+2 r_{2}^{\prime}}-2}{2 e^{2 r_{1}+2 r_{2}+2 r_{2}^{\prime}}+1}, \\
& g_{2}^{o p t}=g_{3}^{o p t}=\frac{e^{2 r_{1}+2 r_{2}+2 r_{2}^{\prime}}+3 e^{2 r_{1}+2 r_{3}+2 r_{3}^{\prime}}-4}{e^{2 r_{1}+2 r_{2}+2 r_{2}^{\prime}}+3 e^{2 r_{1}+2 r_{3}+2 r_{3}^{\prime}}+2} .
\end{aligned}
$$

In 2014, R. Y. Teh and M. D. Reid pointed out the difference between the genuine N-partite entanglement and full $\mathrm{N}$-partite inseparability and presented the criterion inequalities for the genuine multipartite entanglement among optical modes ${ }^{20}$. Based on Ref. [20] and [26], we know that the sum of variances of an observable cannot be less than the weighted s) sum of the variances of the component states for any mixture:

$$
\delta^{2}\left(\hat{S}_{2}\right)+\delta^{2}\left(\hat{S}_{3}\right) \geq \Sigma_{k} P_{k}\left(\delta_{k}^{2}\left(\hat{S}_{2}\right)+\delta_{k}^{2}\left(\hat{S}_{3}\right)\right),
$$

where $P_{k}$ is a probability the system is separable across the bipartition $k$ (thus, $\Sigma_{k} P_{k}=1$ ) and $\delta_{k}^{2}\left(\hat{S}_{2(3)}\right)$ denotes the variance of $\hat{S}_{2(3)}$ for the system in the state $\rho_{k}{ }^{26}$. For tripartite state, since $I_{1}$ is the sum of two variances, we can get 


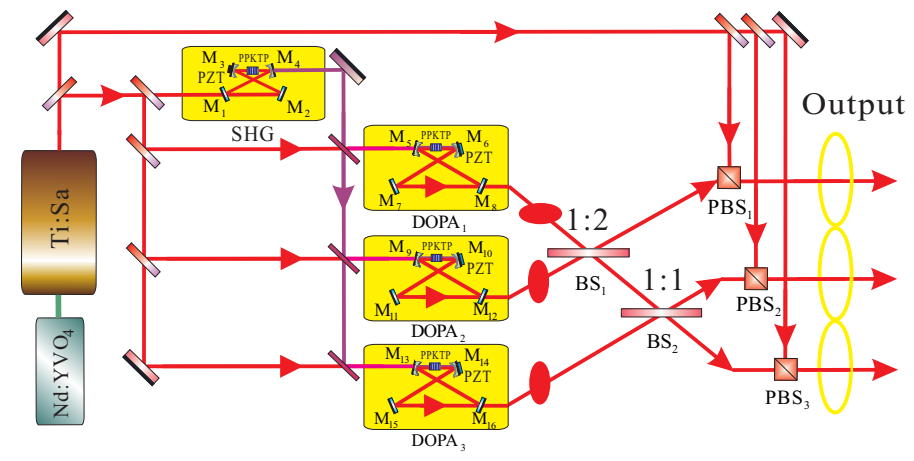

FIG. 2. The experimental setup for the generation of tripartite polarization entangled state. $\mathrm{Nd}: \mathrm{YVO}_{4}: \mathrm{Nd}: \mathrm{YVO}_{4}$ green laser; Ti:Sa, Titanium sapphire laser; SHG: second harmonic generation cavity; DOPA: degenerate optical parameter amplifiers; $\mathrm{BS}_{1-2}$ : beam splitter with different reflectivity; $\mathrm{PBS}_{1-3}$ : polarization beam splitter.

$$
\begin{aligned}
I_{1} & \geq P_{1} I_{1,1}+P_{2} I_{1,2}+P_{3} I_{1,3} \\
& \geq P_{1} I_{1,1}+P_{2} I_{1,2} \geq P_{1}+P_{2} .
\end{aligned}
$$

Similarly, $I_{2} \geq P_{2}+P_{3}$ and $I_{3} \geq P_{3}+P_{1}$. Since $\Sigma_{k} P_{k}=$ 1 , for any mixture it must be true:

$$
I_{1}+I_{2}+I_{3} \geq 2 .
$$

That is, for the genuine tripartite polarization entanglement, the above inequality must be violated ${ }^{20}$.

The experimental setup for the generation of tripartite polarization entanglement is shown in Fig. 2. The Nd: $\mathrm{YVO}_{4}$ green laser (DPSS FG-VIIIB produced by Yuguang company) is used to pump the Titanium sapphire laser (MBR 110 produced by the Coherent company). The output of the Titanium sapphire laser is divided into three parts, the first part is used as the seed beams of three DOPAs, the second part is used as the coherent beam for transferring the quadrature entanglement to the polarization entanglement, the rest is sent to the second harmonic generation (SHG) cavity for obtaining the pump light of three DOPAs. The cavity for SHG is a fourmirror ring cavity consisting of two plane mirrors $\left(\mathrm{M}_{1}, \mathrm{M}_{2}\right)$, two spherical mirrors $\left(\mathrm{M}_{3}, \mathrm{M}_{4}\right)$ and a type-I phase matching $1 \times 2 \times 10 \mathrm{~mm}^{3}$ PPKTP crysta2 ${ }^{27,28}$. The plane mirror $\mathrm{M}_{1}$ is used as the input coupler, which is coated with transmissivity of $13 \%$ at $795 \mathrm{~nm}$. The other three mirrors $\left(\mathrm{M}_{2-4}\right)$ are highly reflecting for subharmonic optical field $(795 \mathrm{~nm})$ and $\mathrm{M}_{4}$ is also coated with anti-reflecting at $397.5 \mathrm{~nm}$ to be the output coupler of the second harmonic optical field. Piezoelectric transducer (PZT) mounted on M3 and Pound-DreverHall technique is used to lock the cavity length. The output beams from SHG with wavelength at $397.5 \mathrm{~nm}$ are split to three parts to pump three DOPAs.

The three DOPAs have the same configuration, which are also the four-mirror ring cavity consisting of two plane mirrors and two spherical mirrors with the radius curvature of $100 \mathrm{~mm}$. A type-I phase matching PPKTP crystal, which is

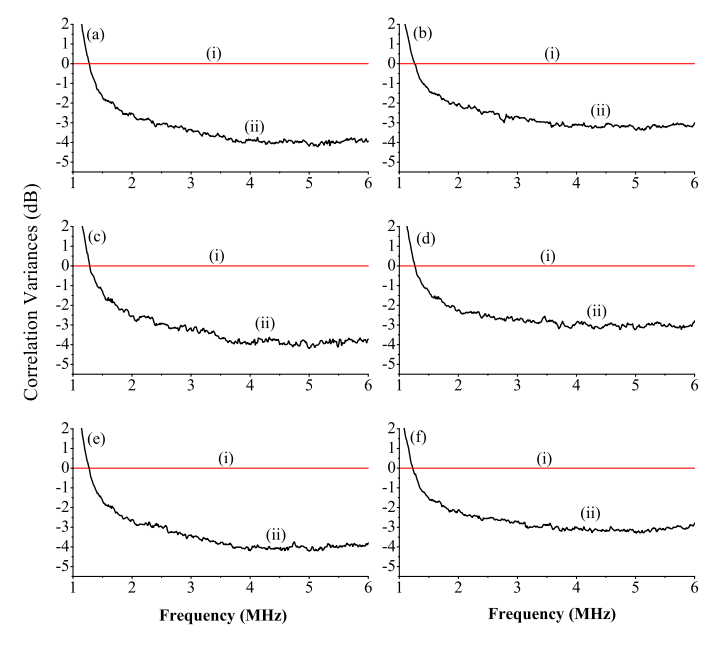

FIG. 3. The measured correlation variances of $\delta^{2}\left(\hat{S}_{2_{d_{2}}}-\hat{S}_{2_{d_{3}}}\right)$ (a), $\delta^{2}\left(g_{1} \hat{S}_{3_{d_{1}}}+\hat{S}_{3_{d_{2}}}+\hat{S}_{3_{d_{3}}}\right)(\mathrm{b}), \delta^{2}\left(\hat{S}_{2_{d_{1}}}-\hat{S}_{2_{d_{3}}}\right)(\mathrm{c}), \delta^{2}\left(\hat{S}_{3_{d_{1}}}+\right.$ $\left.g_{2} \hat{S}_{3_{d_{2}}}+\hat{S}_{3_{d_{3}}}\right)(\mathrm{d}), \delta^{2}\left(\hat{S}_{2_{d_{1}}}-\hat{S}_{2_{d_{2}}}\right)(\mathrm{e})$ and $\delta^{2}\left(\hat{S}_{3_{d_{1}}}+\hat{S}_{3_{d_{2}}}+\right.$ $g_{3} \hat{S}_{3_{d_{3}}}$ ) (f) over the analysis frequency range from 1.0 to $6.0 \mathrm{MHz}$. The trace (i) is the corresponding normalized SNL and the trace (ii) is the quantum correlation noise. The measurement parameters of SA: Resolution Bandwidth (RBW): 300kHz; Video Bandwidth (VBW): $300 \mathrm{~Hz}$.

in the middle of two spherical mirrors, is placed in a coppermade oven and temperature-controlled around $54^{\circ} \mathrm{C}$ with a peltier element for achieving the optimal phase matching. The spherical mirrors $\left(\mathrm{M}_{5}, \mathrm{M}_{9}\right.$ and $\left.\mathrm{M}_{13}\right)$ are used as the input coupler of the corresponding DOPAs, which are coated with anti-reflection for the pump field and high-reflectivity for the subharmonic optical field. Another spherical mirrors $\left(\mathrm{M}_{6}\right.$, $\mathrm{M}_{10}$ and $\left.\mathrm{M}_{14}\right)$ and the plane mirrors $\left(\mathrm{M}_{7}, \mathrm{M}_{11}\right.$ and $\left.\mathrm{M}_{15}\right)$ are coated with high reflection for the subharmonic modes. Another plane mirrors $\left(\mathrm{M}_{8}, \mathrm{M}_{12}\right.$ and $\left.\mathrm{M}_{16}\right)$ coated with $\mathrm{T}=$ $5.0 \%$ for $795 \mathrm{~nm}$ are used as the output couplers. $\mathrm{M}_{6}\left(\mathrm{M}_{10}\right.$, $\mathrm{M}_{14}$ ) is mounted on a PZT to scan actively the cavity length of the $\mathrm{DOPA}_{1}\left(\mathrm{DOPA}_{2}, \mathrm{DOPA}_{3}\right)$ or lock it on resonance with injected seed beam as needed by the Pound-Drever-Hall technique $\frac{7,29}{}$. In order to reduce the threshold and maximize the nonlinear conversion efficiency in DOPAs, a beam waist of $39 \mu \mathrm{m}$ for the subharmonic optical field is chosen by control the length of the cavity. The finesses of $\mathrm{DOPA}_{1}, \mathrm{DOPA}_{2}$ and $\mathrm{DOPA}_{3}$ for the subharmonic mode are 111, 110 and 110, respectively.

When the relative phase between the pump field and seed field is locked to $(2 k+1) \pi(2 k \pi)$ ( $k$ is an integer), the output optical field from DOPAs is quadrature amplitude (phase) squeezed state of light ${ }^{30}$. In our experiment $\mathrm{DOPA}_{1}$ is locked to $2 k \pi$, as well as $\mathrm{DOPA}_{2}$ and $\mathrm{DOPA}_{3}$ are locked to $(2 k+1) \pi$ to obtain the needed quadrature squeezed states as shown in Fig. 1. the power of pump beam for all three DOPAs is about $40 \mathrm{~mW}$, and the power of the seed beam for the DOPA 1 and $\mathrm{DOPA}_{2(3)}$ is about $0.2 \mathrm{~mW}$ and $2 \mathrm{~mW}$ respectively. In this 
case, the power of the output beam from the three DOPAs is almost the same. The quadrature phase squeezed state of light generated by $\mathrm{DOPA}_{1}$ and the quadrature amplitude squeezed state of light generated by $\mathrm{DOPA}_{2}$ are interfered on beam splitter $\mathrm{BS}_{1}$. One of two output beams from $\mathrm{BS}_{1}$ and the output of $\mathrm{DOPA}_{3}$ are interfered on $\mathrm{BS}_{2}$. The relative phase between the two input beams of $\mathrm{BS}_{1}$ and $\mathrm{BS}_{2}$ is $2 k \pi$. The outcoming three optical beams from the two beam splitters are in a tripartite GHZ-like entangled states. Then, the obtained tripartite quadrature entangled states are transformed into tripartite polarization entanglement by coupling with three strong coherent beams on $\mathrm{PBS}_{1-3}$ with the phase difference of $2 k \pi$. The three output beams are detected by three sets of Stokes parameters measurement systems with a Spectrum analyzer (SA), which have been introduced in the previous experiment ${ }^{13}$. The output from DOPA is a broadband quadrature squeezed state of light, i.e. we can observe the squeezing phenomenon within the frequency bandwidth of the DOPA. Since in the region of lower frequencies the quantum noise of the laser is far higher than the shot noise limit (SNL) due to the influence of the extra noises in the pump laser, we measure the correlation variances over the analysis frequency range from 1.0 to $6.0 \mathrm{MHz}$. Figs. 3 show the measured correlation variances of $\delta^{2}\left(\hat{S}_{2_{d_{2}}}-\hat{S}_{2_{d_{3}}}\right), \delta^{2}\left(g_{1} \hat{S}_{3_{d_{1}}}+\hat{S}_{3_{d_{2}}}+\hat{S}_{3_{d_{3}}}\right)$, $\delta^{2}\left(\hat{S}_{2_{d_{1}}}-\hat{S}_{2_{d_{3}}}\right), \delta^{2}\left(\hat{S}_{3_{d_{1}}}+g_{2} \hat{S}_{3_{d_{2}}}+\hat{S}_{3_{d_{3}}}\right), \delta^{2}\left(\hat{S}_{2_{d_{1}}}-\hat{S}_{2_{d_{2}}}\right)$ and $\delta^{2}\left(\hat{S}_{3_{d_{1}}}+\hat{S}_{3_{d_{2}}}+g_{3} \hat{S}_{3_{d_{3}}}\right)$, respectively. The traces (ii) are the measured quantum correlation noises and the traces (i) are the corresponding normalized SNL. When the correlation variances of $\delta^{2}\left(g_{1} \hat{S}_{3_{d_{1}}}+\hat{S}_{3_{d_{2}}}+\hat{S}_{3_{d_{3}}}\right), \delta^{2}\left(\hat{S}_{3_{d_{1}}}+g_{2} \hat{S}_{3_{d_{2}}}+\right.$ $\left.\hat{S}_{3_{d_{3}}}\right)$ and $\delta^{2}\left(\hat{S}_{3_{d_{1}}}+\hat{S}_{3_{d_{2}}}+g_{3} \hat{S}_{3_{d_{3}}}\right)$ are measured, the optimal gains are chosen to minimize the correlation variances for maximizing the measured entanglement. The experimentally optimal gains are in good agreement with the theoretically calculated values $\left(g_{1}^{o p t}=g_{2}^{o p t}=g_{3}^{o p t}=0.845\right)$ from Eqs. (6-7). Both of the correlation variances are below the corresponding SNL throughout the frequency range from $1.3 \mathrm{MHz}$ to 6.0 MHz. The best entanglement is observed at $5 \mathrm{MHz}$ with $I_{1}=0.42 \pm 0.08, I_{2}=0.41 \pm 0.08, I_{3}=0.42 \pm 0.08$ and $I_{1}+I_{2}+I_{3}=1.25 \pm 0.07$, which violate the criteria for both tripartite inseparability and genuine tripartite entanglement, thus we say that the genuine tripartite polarization entanglement is verified.

To the conclusion, we present the experimental generation of $\mathrm{CV}$ multipartite polarization entanglement by means of transforming the quadrature entanglement into a polarization basis. In the presented scheme, multipartite quadrature entangled states are the basic sources for the generation of multipartite polarization entangled states. Although we just combined a tripartite entangled state and three bright coherent optical beams to produce a tripartite polarization entangled state in this experiment, the entangled states with much more submodes are possible to be produced if the multipartite quadrature entangled states are available. Using the quadrature entangled states involving more submodes 8,31 and a proper beamsplitter network, the presented method can be extended to prepare polarization entangled states with more submodes and thus has potential applications in the future quantum information networks.

We acknowledge the support from the Natural Science Foundation of China (Grants Nos. 11322440, 11474190, 11304190,11504220), FOK YING TUNG Education Foundation, Natural Science Foundation of Shanxi Province (Grant No. 2014021001).

${ }^{1}$ S. L. Braunstein, and P. van Loock, Rev. Mod. Phys. 77, 513 (2005).

${ }^{2}$ D. Bouwmeester, J. W. Pan, K. Mattle, M. Eibl, H. Weinfurter, and A. Zeilinger, Nature 390, 575, (1997).

${ }^{3}$ D. Boschi, S. Branca, F. DeMartini, L. Hardy, and S. Popescu, Phys. Rev. Lett. 80, 1121 (1998).

${ }^{4}$ K. Honda, D. Akamatsu, M. Arikawa, Y. Yokoi, K. Akiba, S. Nagatsuka, T. Tanimura, A. Furusawa, and M. Kozuma, Phys. Rev. Lett. 100, 093601 (2008).

${ }^{5}$ J. Appel, E. Figueroa, D. Korystov, M. Lobino, and A. I. Lvovsky, Phys. Rev. Lett. 100, 093602 (2008).

${ }^{6}$ K. Jensen, W. Wasilewski, H. Krauter, T. Fernholz, B. M. Nielsen, M. Owari, M. B. Plenio, A. Serafini, M. M. Wolf, and E. S. Polzik, Nat. Phys. 7, 13 (2010).

${ }^{7}$ X. J. Jia, J. Zhang, Y. Wang, Y. P. Zhao, C. D. Xie, and K. C. Peng, Phys. Rev. Lett. 108, 190501 (2012).

${ }^{8}$ X. L. Su, S. H. Hao, X. W. Deng, L. Y. Ma, M. H. Wang, X. J. Jia, C. D. Xie, and K. C. Peng, Nat. Commun. 4, 2828 (2013).

${ }^{9}$ H. J. Kimble, Nature 453, 1023 (2008).

${ }^{10}$ X. J. Jia, Z. H. Yan, Z. Y. Duan, X. L. Su, H. Wang, C. D. Xie, and K. C. Peng, Phys. Rev. Lett. 109, 253604 (2012).

${ }^{11}$ L. C. Chen, Z. M. Meng, and X. D. Yu, Acta Sinica Quantum Optica 20, 119 (2014).

${ }^{12}$ B. Julsgaard, A. Kozhekin, and E. S. Polzik, Nature 413, 400 (2001).

${ }^{13}$ W. P. Bowen, N. Treps, R. Schnabel, and P. K. Lam, Phys. Rev. Lett. 89, 253601 (2002).

${ }^{14}$ O. Glöckl, J. Heersink, N. Korolkova, G. Leuchs, and S. Lorenz, J. Opt. B: Quantum Semiclass. Opt. 5, S492 (2003).

${ }^{15}$ T. Sh. Iskhakov, I. N. Agafonov, M. V. Chekhova, and G. Leuchs, Phys. Rev. Lett. 109, 150502 (2012).

${ }^{16}$ N. Korolkova, G. Leuchs, R. Loudon, T. C. Ralph, and C. Silberhorn, Phys. Rev. A 65, 052306 (2002).

${ }^{17}$ R. F. Dong, J. Heersink, J. Yoshikawa, O. Glöckl, U. L Andersenand, and G. Leuchs, New J. of Phys. 9, 410, (2007).

${ }^{18}$ V. Josse, A. Dantan, A. Bramati, M. Pinard, and E. Giacobino, Phys. Rev. Lett. 92, 123601 (2004).

${ }^{19}$ Z. H. Yan, and X. J. Jia, J. Opt. Soc. Am. B 32, 2139 (2015).

${ }^{20}$ R. Y. Teh, and M. D. Reid, Phys. Rev. A 90, 062337 (2014).

${ }^{21}$ G. G. Stokes, Trans. Camb. Phil. Soc. 9, 399 (1852).

${ }^{22}$ P. van Loock, and A. Furusawa, Phys. Rev. A 67, 052315 (2003).

${ }^{23}$ J. Zhang, C. D. Xie, K. C. Peng, Phys. Lett. A 299, 427 (2003).

${ }^{24}$ L.M. Duan, G. Giedke, J. I. Cirac, and P. Zoller, Phys. Rev. Lett. 84, 2722 (2000).

${ }^{25}$ R. Simon, Phys. Rev. Lett. 84, 2726 (2000).

${ }^{26}$ H. F. Hofmann, and S. Takeuchi, Phys. Rev. A 68, 032103 (2003).

${ }^{27}$ X. Wen, Y. S. Han, J. D. Bai, J. He, Y. H. Wang, B. D. Yang, and J. M. Wang, Opt. Express 22, 32293 (2014).

${ }^{28}$ X. Deng, J. Zhang, Y. C. Zhang, G. Li, and T. C. Zhang, Opt. Express 21, 25907 (2013).

${ }^{29}$ Y. Y. Zhou, X. J. Jia, F. Li, C. D. Xie, and K. C. Peng, Opt. Express 23, 4952 (2015).

${ }^{30}$ M. Mehmet, S. Ast, T. Eberle, S. Steinlechner, H. Vahlbruch, and R. Schnabel, Opt. Express 19, 25763 (2011).

${ }^{31}$ M. Yukawa, R. Ukai, P. van Loock, and A. Furusawa, Phys. Rev. A 78, 012301 (2008). 